\title{
Habilidades de argumentación. Una propuesta para el planteamiento de posibles soluciones a los conflictos interpersonales ${ }^{*}$
}

\author{
Argumentative abilities. A proposed approach \\ to possible solutions in interpersonal conflicts
}

\section{Habilidades de argumentação. Uma proposta para a abordagem de possíveis soluções aos conflitos interpessoais}

\author{
Angélica María Rodríguez Ortiz** \\ Rosa Elmira Coral Cadena*** \\ María Guadalupe Andino**** \\ Óscar William Portilla****
}

Fecha de recepción: 31 de julio 2017

Fecha de evaluación: 5 de septiembre de 2017

Fecha de aceptación: 5 de octubre de 2017

Fecha de publicacion en linea: 1 de diciembre de 2017

DOI: http://dx.doi.org/10.18359/reds.2966

Cómo citar este artículo:

Rodríguez Ortiz, A.M., Coral Cadena, R.E., Andino, M.G. y Portilla, O.W. (2017). Habilidades de argumentación. Una propuesta para el planteamiento de posibles soluciones a los conflictos interpersonales. Revista Educación y Desarrollo Social, 11(2), 32-54. DOI: org/10/18359/reds.2966.

\footnotetext{
Artículo derivado de la investigación Habilidades para la argumentación. Una propuesta para el planteamiento de posibles soluciones a los conflictos interpersonales que presentan los estudiantes de grado quinto de primaria en el entorno escolar, de la Institución Educativa Rural Francisco José de Caldas en la Inspección Siberia, municipio de Orito Putumayo, Universidad Autónoma de Manizales (2017); adscrito al grupo de investigación Educación y Cognición y al grupo SEAD-UAM, en la línea Actores y Contextos.

* Doctora en Filosofía, Universidad Pontificia Bolivariana; Magíster en Educación, Universidad de Caldas, y Licenciada en Filosofía y Letras. Líder y docente del programa de Maestría en Enseñanza de las Ciencias, Universidad Autónoma de Manizales, modalidad a distancia. Investigadora del grupo SEAD-UAM en la línea de actores y contextos. ORCID: http://orcid.org/0000-0002-7710-9915. Correo electrónico: angelica.rodriguez276@gmail.com, amrodriguez@autonoma.edu.co

*** Magíster en Enseñanza de las Ciencias, Universidad Autónoma de Manizales; especialista en Pedagogía de la Recreación Ecológica y Licenciada en Educación Básica con énfasis en Ciencias Naturales y Educación Ambiental. Docente de la Institución Educativa Rural Francisco José de Caldas de la Inspección Siberia, Orito, Putumayo. ORCID: http://orcid.org/0000-0001-7212-4684. Correo electrónico: rosacoral78@gmail.com

**** Magíster en Enseñanza de las Ciencias, Universidad Autónoma de Manizales (2017). Licenciada en Educación Básica Primaria, Universidad Mariana (2004). Docente de la IER Francisco José de Caldas, ubicada en la Inspección de Siberia, municipio de Orito, Putumayo. ORCID: http://orcid.org/0000-

32 0002-0584-3125. Correo electrónico: marilup1973@gmail.com. Docente de la I.E.R. Francisco José de Caldas.

***** Magíster en enseñanza de las Ciencias, Universidad Autónoma de Manizales. Docente de la Institución Educativa Rural Francisco José de Caldas de la Inspección Siberia, Orito, Putumayo. Correo electrónico: oswipo@hotmail.es
} 
Habilidades de argumentación. Una propuesta para el planteamiento de posibles soluciones...

\title{
Resumen
}

El presente artículo muestra los resultados de una investigación en torno a la influencia del desarrollo de habilidades argumentativas en el planteamiento de posibles soluciones a los conflictos interpersonales que se presentan en el entorno escolar. Se realizó un estudio cualitativo descriptivo, en el cual se diseñó una unidad didáctica desde la estrategia del análisis de dilemas morales, a partir de situaciones conflictivas presentadas en el aula — adaptados al modelo de desarrollo moral de Kohlberg- El estudio permitió la comprensión del concepto de conflicto interpersonal y el uso de la argumentación para el planteamiento de posibles soluciones sustentadas en argumentos razonados y razonables. La investigación permitió concluir que el desarrollo de habilidades argumentativas les brinda a los estudiantes herramientas conceptuales y metodológicas para afrontar situaciones de conflicto interpersonal de forma constructiva, de manera que se planteen soluciones argumentadas.

Palabras clave: argumentación, conflicto interpersonal, desarrollo moral, razonado y razonable.

\begin{abstract}
This article shows the results of an investigation about the influence of the development of argumentative abilities on the approach of possible solutions to the interpersonal conflicts that occur in the school environment. A descriptive qualitative study was carried out, in which a didactic unit was designed, including the analysis of moral dilemmas of conflictive situations presented in the classroom as a strategy-adapted to the Kohlberg model-to emerge the students' speeches. A descriptive qualitative study was carried out, in which a didactic unit was designed following the strategy of moral dilemma analysis, based on conflictive situations presented in the classroom-adapted to Kohlberg's moral development model. This study allowed understanding of the concept of interpersonal conflict and the use of argumentation to approach possible solutions based on reasoned and reasonable arguments. This research concluded that the development of argumentative abilities provides students with conceptual and methodological tools, in order to deal with situations of interpersonal conflict constructively in a way that argumentative solutions arise.
\end{abstract}

Keywords: Argumentation, interpersonal conflict, moral development, reasoned and reasonable.

\section{Resumo}

O presente artigo mostra os resultados de uma investigação sobre a influência do desenvolvimento de habilidades argumentativas na abordagem de possíveis soluções aos conflitos interpessoais que são apresentados no ambiente escolar. Foi realizado um estudo qualitativo descritivo, no qual foi projetada uma unidade didática a partir da estratégia de análise de dilemas morais, baseada em situações conflitantes apresentadas na sala de aula — adaptada ao modelo de desenvolvimento moral de Kohlberg- O estudo permitiu a compreensão do conceito de conflito interpessoal e o uso da argumentação para a apresentação de possíveis soluções baseadas em argumentos razoados e razoáveis. A pesquisa permitiu concluir que o desenvolvimento de habilidades argumentativas proporciona aos alunos ferramentas conceituais e metodológicas para lidar com situações de conflito interpessoal de forma construtiva, de maneira a que surjam soluções argumentadas.

Palavras-chave: argumentação, conflito interpessoal, desenvolvimento moral, razoado e razoável. 
$\overline{\text { Angélica María Rodríguez Ortiz, Rosa Elmira Coral Cadena, María Guadalupe Andino, Óscar William Portilla }}$

\section{Introducción}

La argumentación es la clave para la comunicación, ya que fortalece la manera de formular y justificar puntos de vista en la construcción de argumentos en el proceso social. La escuela es un escenario donde los niños interactúan; allí empiezan a construir nuevas redes sociales donde se pone de manifiesto la ausencia de la competencia argumentativa al momento de enfrentarse a situaciones disruptivas. La falta de herramientas para afrontar este tipo de situaciones que afectan las relaciones interpersonales y el ambiente en el aula repercuten en el desarrollo cognitivo y social. Actualmente, muchos estudios han centrado sus procesos de investigación en el desarrollo de la argumentación en las aulas de clase en el plano de la resolución de conflictos interpersonales, con el afán de entender este fenómeno y poder afrontarlos de forma apropiada.

La enseñanza de la actividad discursiva argumentativa es compleja. Los estudiantes presentan dificultades al momento de expresar y organizar sus ideas en un argumento razonado que se identifique, desde el punto de vista lógico, por su coherencia y su estructuración. Una de las tareas en la práctica educativa es incluir procesos didácticos que permitan orientar a los estudiantes hacia el desarrollo de habilidades que hagan posible la gestión de conflictos a partir de la argumentación, para plantear alternativas razonadas y razonables que regulen de forma coherente sus argumentos. Es destacable, que

El problema no radica en el conflicto en sí, sino en su manejo inadecuado. En este caso, es necesario que la escuela cuente con acciones concertadas para su manejo, lo cual se convierte en un reto para que la comunidad educativa desarrolle estrategias que le permitan resolver los conflictos de manera adecuada y construir aprendizajes a partir de lo ocurrido. (Ministerio de Educación Nacional de Colombia, 2014, p. 25).

De ahí que el hecho de desarrollar habilidades destinadas a generar procesos argumentativos que desarrollen la capacidad de expresar los planteamientos de forma razonada y razonable a partir del reconocimiento de los puntos de vista disidentes, la disertación y el consenso entre sus pares posibilite un proceso básico de pensamiento que favorece el razonamiento y promueve habilidades comunicativas que son fundamentales para hacer frente a los conflictos de forma constructiva. Para Haba (1978), las soluciones a los conflictos deben "[...] resultar de una discusión, un diálogo, una argumentación, en donde las distintas opiniones tengan la oportunidad de salir a la luz y ser confrontadas entre sí" (p. 21). Por ello, puede decirse que la argumentación es una estrategia que favorece el diálogo que admite posiciones a favor o en contra de una tesis que sostiene el autor. 
Lograr que los estudiantes expresen argumentos razonables que pongan en consideración y sean aceptados por sus congéneres requiere un nivel de desarrollo moral que permita al sujeto el reconocimiento de principios convencionales. Para Kohlberg (1992) "[...] el foco está en mantener el orden social obedeciendo la ley y cumpliendo con el deber" (p. 374) para que estos conduzcan al sujeto a una toma de decisiones más equitativa, que se reafirme con razones en las que se ponga en consideración el bienestar colectivo. Es necesario pensar en una educación moral porque "[...] de acuerdo con las etapas de desarrollo surgen diferencias en la forma de pensar de los niños de edades diferentes. Así mismo, también se manifiestan diferencias en las formas de actuar" (Rodríguez y Hernández, 2016, p. 35).

En definitiva, se considera relevante contribuir a la reflexión en torno a cómo la argumentación permite plantear soluciones razonadas a los conflictos interpersonales que se presentan en el entorno escolar, ya que a través de la aplicación de la unidad didáctica se forma al estudiante en el ejercicio de la autonomía.

\section{Marco teórico: la argumentación en la escuela}

La argumentación —como capacidad discursiva para defender las ideas con un conjunto de argumentos, con la cual busca persuadir a otros y aceptar las diferencias de los demás - implica com- prender lo que está implícito. Se considera importante desarrollar la argumentación a partir de la teoría de Toulmin (2007), entendiendo a los estudiantes como sujetos que pueden resolver sus conflictos interpersonales de forma constructiva a través de la palabra, promoviendo su formación crítica; es decir, sujetos capaces de justificar su propia posición. Según Camps y Dolz (1995), saber argumentar constituye, "[...] para todos los actores de una democracia, el medio fundamental para defender sus ideas, para examinar de manera crítica las ideas de los otros, para rebatir los argumentos de mala fe y para resolver muchos conflictos de intereses" (p. 7).

La argumentación es un proceso natural de carácter persuasivo; es el intento de dar razones que avalen las afirmaciones (hechos, opiniones y creencias) con justificaciones sólidas y bien estructuradas para convertirlas en conclusiones convincentes. "Para Toulmin (1958), la argumentación se refiere a la capacidad de exponer una tesis, controvertirla, examinar sus consecuencias, intercambiar pruebas y plantear buenas razones que lleven a una conclusión" (Monsalve Upegui, 1992, p. 217). Los argumentos como construcciones intelectuales son fruto de procesos y estructuras que, según Toulmin (2007), permiten un análisis de sus elementos y, de este modo, afirmar con un grado de certeza la fuerza y el carácter concluyente de los argumentos. 
$\overline{\text { Angélica María Rodríguez Ortiz, Rosa Elmira Coral Cadena, María Guadalupe Andino, Óscar William Portilla }}$

La argumentación como posibilidad de abordar los conflictos interpersonales

Una problemática que se vive en el aula escolar es el conflicto interpersonal, considerado este último como una situación socialmente relevante sobre la cual se debe hacer énfasis en el aula de clase. Por lo anterior, se plantea una unidad didáctica estructurada con base en análisis de dilemas morales que contribuyan a que los estudiantes desarrollen habilidades para la argumentación en el planteamiento de posibles soluciones a los conflictos interpersonales que se presentan entre compañeros en el contexto escolar. Saber abordar los conflictos implica aprender a trabajar con el otro, ser capaz de discernir y enfrentar con racionalidad moral el conflicto. Es menester orientar en habilidades para la argumentación —en especial el análisis, la síntesis y la reflexión-.

La capacidad para manejar conflictos, pacífica y constructivamente, requiere de ciertos conocimientos sobre las dinámicas de los conflictos, de competencias cognitivas como la capacidad de generar opciones creativas ante una situación de conflicto [...] de competencias comunicativas como la capacidad de transmitir asertivamente intereses particulares, teniendo cuidado de no agredir a los demás. (Ruiz y Chaux, 2005, p. 45)

La habilidad de análisis permite al estudiante hacer una lectura de la situación conflictiva para examinar sus elementos y entender la relación que se da entre estos; la habilidad de síntesis, por su parte, ayuda a reconstruir el hecho para el procesamiento de la información y llegar a una comprensión que permita emitir una conclusión argumentada. Por último, la habilidad de reflexión lleva al estudiante a realizar una acción metacognitiva sobre su forma de actuar y a sustentar posturas para tomar una decisión con argumentos razonados y razonables.

\section{La argumentación como estrategia para plantear posibles soluciones a los conflictos interpersonales}

La argumentación es una herramienta esencial en la gestión de alternativas para el abordaje de los conflictos. Es un proceso básico de pensamiento que favorece el razonamiento y promueve el desarrollo de habilidades comunicativas que son fundamentales para hacer frente a los conflictos de forma constructiva. La argumentación faculta al estudiante para dar razones bien sustentadas ante un conflicto; le permite explicar y defender a partir de argumentos por qué se debe actuar de una u otra manera. A su vez, le ayuda, a través de la reflexión, a ceder frente a los argumentos del otro después de analizarlos y evaluarlos críticamente.

La argumentación es primordial para intervenir el conflicto y gestionar alternativas razonadas y razonables para transformarlo, porque "[...] argumentar promueve el desarrollo [...] de competencias ciudadanas" (Sánchez, González y García, 2013, p. 16). Desde esta línea 
de pensamiento, es esencial mejorar en el aula los procesos de interacción comunicativa que se expresan frente a un conflicto en la discusión, el análisis, el disenso y la reciprocidad para consolidar procesos comunicativos mediados por el diálogo entre sus pares; por ello, se propone fortalecer la competencia argumentativa para que los estudiantes puedan identificar los elementos básicos de estos y considerarlos al tomar y defender una posición.

Un planteamiento razonado ${ }^{1}$ "[... e es la cadena ilativa que, orientada hacia una finalidad de conocimiento (teórico o práctico), conduce de una premisa inicial (sea un principio, sea una consecuencia), aceptada de antemano, hacia una conclusión final (principio o consecuencia)" (Haba, 1978, p. 5). Esto implica que los argumentos sean lógicos y coherentes, lo cual involucra tomar como base la estructura argumentativa sustentada en tres elementos, partes o momentos que propone Toulmin (2007), en los que se están implícitas las razones, las garantías y las conclusiones.

La razón de la cual se extrae la información es "como una premisa singular, transmite la información a partir de la cual se extrae una conclusión" (Toulmin, 2007, p. 154). La garantía, por otra parte, legitima la conclusión; es la "premisa universal, expresa, no algún tipo de información, sino una garantía o justificación de acuerdo con la cual el hablante puede pasar con seguridad del dato a la conclusión" (Toulmin, 2007, p. 154). Por último, la conclusión es el argumento final, dotado de coherencia y cohesión. "La conclusión es una mera reformulación del enunciado inicial [...] que ya ha sido afirmado implícitamente en los datos o en respaldo de nuestra idea" (Toulmin, 2007, p. 167). Estos aportes intervienen en un modelo argumentativo propicio para dar firmeza a sus argumentos ${ }^{1}$.

Los argumentos son razonables cuando la persona, al momento de emitirlos, es consciente de las consecuencias que trae consigo cada una de las premisas. "Los argumentos manejados son razonables en la medida, justamente, en aquellos que los sostienen apelan a un reconocimiento virtual de su plausibilidad por parte de todo el mundo o por lo menos de cualquier ciudadano razonable" (Haba, 1978, p. 22).

Así pues, es necesario que los estudiantes adquieran estas habilidades para que puedan relacionarse con el otro; que aprendan a discernir los puntos de

\footnotetext{
Es preciso aclarar que esta estructura argumentativa no es la única dentro del campo de la argumentación, aunque sí es la más sencilla y propicia para ejercitar inicialmente al estudiante en el plano argumentativo. Perfectamente algunos argumentos podrían basarse solo en premisas singulares (v. gr., los argumentos inductivos) (Perelman y Olbrechts-Tyteca, 1989, § 78) o solo en generales (como los hipotéticos o probables) (§ 59) para llegar a una conclusión, y, a su vez, podrían darse argumentos en los que no necesariamente dentro de la conclusión deben estar implícitas todas las premisas (v.gr., : la abducción o un argumento pragmático) (\$ 62).
} 
$\overline{\text { Angélica María Rodríguez Ortiz, Rosa Elmira Coral Cadena, María Guadalupe Andino, Óscar William Portilla }}$

vista de sus congéneres para llegar a un consenso de manera que transformen el conflicto constructivamente.

\section{El conflicto interpersonal desde una perspectiva constructiva}

El conflicto interpersonal es una situación inherente al ser humano; se presenta en un contexto de interacción social que se manifiesta de acuerdo con la manera como se aborden. Ugalde (2009) afirma que los conflictos asumidos desde una postura positiva pueden ser aprovechados para el desarrollo de la comunicación asertiva, la cual favorece la argumentación y potencia las habilidades sociales. Por tal razón, algunas concepciones permiten hacer una aproximación a su naturaleza y a la relevancia que se le otorga para vincularlo en los procesos de enseñanza y aprendizaje en el aula escolar. Cortina (1997) define el conflicto así:

Una situación de enfrentamiento provocada por una contraposición de intereses — sea real o aparente-en relación con un mismo asunto; esta situación puede producir verdadera angustia en las personas normalmente constituidas cuando no se vislumbra una salida satisfactoria y el asunto es importante para ellas. (p. 54)

El conflicto se entiende como una situación contradictoria en la que se enfrentan las personas con el objeto de satisfacer sus intereses; algunos se manifiestan al expresarse en conductas disruptivas cuando no se afronta de forma adecuada.
Para Migdalek, Santibáñez y Rosemberg (2014) es fundamental que desde temprana edad se oriente a los niños en el manejo de conflictos a través de la argumentación para que sean ellos los que puedan tomar decisiones morales asertivamente. "Así, entendemos que el conflicto es una realidad social que no deberíamos pretender abolir o prohibir, sino más bien buscarle salidas positivas" (Fundación UNIR Bolivia, s. f., p. 11).

Durante el proceso de interacción se puede observar cómo se exteriorizan los conflictos a través de manifestaciones verbales o físicas, pero a veces permanecen latentes, lo que provoca en sus pares una modificación grave en sus comportamientos; esto se da por la falta de la capacidad de expresar sus puntos de vista justificándolos.

Es relevante reconocer las actitudes, el comportamiento y la contradicción como aspectos esenciales de un conflicto para abordar de forma adecuada una situación conflictiva. El conflicto "[...] puede enfocarse básicamente como una de las fuerzas motivadoras de nuestra existencia, como una causa, un concomitante y una consecuencia del cambio, como un elemento tan necesario para la vida social como el aire para la vida humana (Galtung, 1981, p. 11).

Para Jares (1997) el conflicto trae consigo aspectos positivos y negativos que pueden hacer que un grupo, según las circunstancias en las que se desarrolle la situación conflictiva, permita el cambio en los procesos de interacción 
social. Cuando no se interviene de forma adecuada se expresarán en conductas antagónicas que afectan las relaciones y dificultan los consensos y los acuerdos. Para saber manejar un conflicto es necesario entender este fenómeno desde su naturaleza y elementos como los protagonistas (las causas y las consecuencias), y a partir de este conocimiento ampliar su comprensión fortaleciendo procesos argumentativos. Es necesario "[...] analizar y comprender la naturaleza, estructura y elementos que componen todo conflicto, y a partir de ello detallar, aprender, y practicar unos métodos, no para eliminar el conflicto, sino para regularlo y encausarlo hacia resultados positivos" (Lederach, 1993, p. 137).

En este sentido, el proceso de transformación de un conflicto es prioritario en función del logro de los aprendizajes en los involucrados; asimismo, posibilita la construcción de procesos para la comprensión de fenómenos sociales.

\section{La unidad didáctica}

La unidad didáctica Los conflictos interpersonales como oportunidad de aprendizaje para convivir en la escuela responde a la necesidad de detectar la forma como conciben los estudiantes el conflicto interpersonal e identificar la calidad de los argumentos. "Se considera que los objetivos que orientan el diseño de una unidad didáctica para la enseñanza de las ciencias deberían basarse en concretar cuáles son las dificultades y obstáculos que se pretende ayudar a superar" (Peterfalvi, citado en Perales y Cañal de León, 2015, p. 5). Se pretende que, desde los conocimientos previos de los estudiantes, se concreten las dificultades y los obstáculos que se presentan, para desarrollar la argumentación y superar la comprensión del concepto.

La unidad didáctica consta de tres fases. Las actividades se presentan a través del planteamiento de dilemas morales adaptados en el modelo de Kohlberg (1992) y se involucra el uso de las tecnologías de la información y la comunicación (TIC) - como la construcción de historietas animadas, usando el programa Toondoo y Go Animate-, que involucran el análisis de situaciones de conflicto que se exteriorizan en el entorno escolar, a fin de que identifiquen los elementos estructurales del conflicto y refieran un fundamento conceptual para tomar posturas críticas frente a un planteamiento de forma lógica y sustentada con argumentos razonados y razonables a través de reflexiones metacognitivas (véase figura 1).

\section{Los dilemas morales como estra- tegia en la unidad didáctica}

Emitir argumentos en los que se consideren algunos fundamentos para que sean generalmente aceptados por los demás requiere, según Kohlberg (1992), un nivel de desarrollo moral que permita al sujeto el reconocimiento de principios convencionales. Bascón, De la Mata y Cala (2006) y Alzate y Henao (2012) han señalado que analizar la argumentación de los adolescentes ante la resolución de conflictos haciendo uso de dilemas 


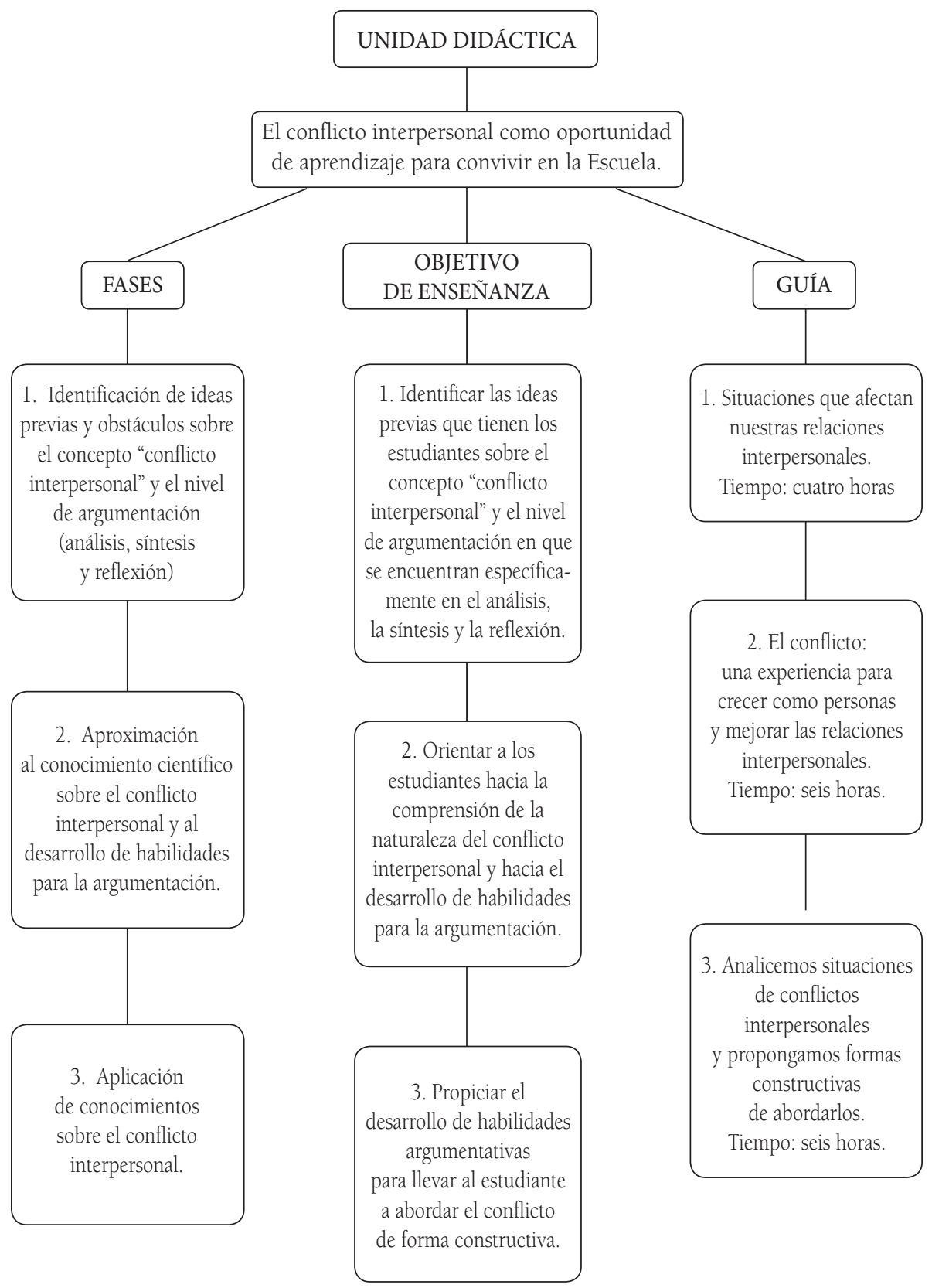

Figura 1. Estructura de unidad didáctica

Fuente: elaboración propia. 
morales - como propiciadores de la controversia y la posibilidad de negociación- permite generar en el estudiante la reflexión de forma autónoma, crítica y responsable de decisiones justas ante un conflicto interpersonal. Bermúdez y Jaramillo (2000) definen el dilema como:

Una breve historia sobre un personaje que enfrenta una situación difícil y tiene que tomar una decisión sobre la mejor acción a seguir. Aquella que tome involucra aspectos importantes de su vida, pero también puede afectar a otras personas. Por esta razón, el personaje debe tomar una decisión que sea buena, correcta o justa consigo mismo y con los demás que se verán afectados por ella; de allí que deba enfrentarse a una decisión moral. (p. 35)

El uso del análisis de dilemas morales como estrategia para que el estudiante se posesione en el papel de los protagonistas, y que de esta forma él asuma posturas razonadas para abordar la situación planteada, permite el desarrollo de las habilidades para la argumentación que fortalece, a su vez, la formulación y justificación de puntos de vista en la construcción de argumentos para estar en capacidad de abordar la resolución de conflictos de manera constructiva.

\section{Metodología}

Esta es una investigación de tipo cualitativo y sigue un método etnográfico, y a través de estos se hace una descripción detallada del tema a investigar para llegar a la comprensión de la realidad que viven los estudiantes, relacionándola con los argumentos que formulan al momento de abordar un conflicto interpersonal. Con base en esto, se planteó como técnica la unidad didáctica y se pretendió dar a conocer los argumentos de los estudiantes para el planteamiento de posibles soluciones. Esta unidad didáctica se trabajó desde un enfoque hermenéutico, porque a partir de este se llega a una interpretación y comprensión de las actitudes conflictivas que manifiestan los estudiantes de grado quinto y, teniendo en cuenta los resultados, se puede producir un conocimiento que ayude a superar las debilidades que tienen. Con la unidad se busca un acercamiento crítico, reflexivo y transformador de la realidad social.

A partir del problema que se aborda en la investigación y con base en los objetivos planteados, se establecen las categorías de análisis, que están estrechamente relacionadas con las habilidades propias de la argumentación (específicamente, el análisis, la síntesis y la reflexión). También se tienen en cuenta los modelos argumentativos de Toulmin (2007) y, además, para la conceptualización del conflicto interpersonal — como categoría emergente que surge del proceso de la aplicación de la unidad didáctica一, los niveles de desarrollo moral de Kohlberg (1992). 
$\overline{\text { Angélica María Rodríguez Ortiz, Rosa Elmira Coral Cadena, María Guadalupe Andino, Óscar William Portilla }}$

En el proceso de análisis se identificaron los argumentos de los estudiantes para configurar una codificación que luego permite, por la recurrencia y relevancia de aparición, clasificarlos por categorías y establecer relaciones a través de la triangulación temporal, así como construir las interpretaciones fundamentadas entre los datos y los presupuestos teóricos expuestos en las diferentes investigaciones realizadas en este campo.

\section{Resultados y discusión}

Los resultados que se muestran a continuación se obtuvieron a partir del análisis de las fases planteadas en la unidad didáctica, relacionándolas con las categorías de investigación.

\section{Habilidades para la argumentación}

La comparación de los discursos argumentativos de los estudiantes llevó a identificar el nivel de análisis, síntesis y reflexión que estos presentaron. Paralelo a esto, se fueron identificando los modelos argumentativos que usaron; por tanto, en la estructura de los argumentos de los estudiantes se buscó identificar las razones, garantías y conclusiones implícitas en sus planteamientos sobre la comprensión del concepto conflicto interpersonal y su abordaje. Para determinar el nivel de análisis, síntesis y reflexión en el cual se encontraban los estudiantes se establecieron unos indicadores que orientaron el proceso de análisis de estas subcategorías. Tomando como base estos criterios, se analizaron los discursos de los estudiantes y se ubicaron en determinado nivel (véase tabla 1).

En el análisis realizado de algunos de los datos se pudo evidenciar que en la fase uno los estudiantes se encuentran en un nivel inicial bajo, ya que presentan dificultades y obstáculos en el nivel de argumentación (análisis, síntesis y reflexión). Por ejemplo, el estudiante E12, al presentarle una historieta con una situación conflictiva y preguntarle "iqué opinas sobre la forma como abordaron la situación los personajes? y "si tú estuvieras en el lugar de alguno de los involucrados, ¿cómo actuarías y por qué actuarías así?" respondió lo siguiente: "la forma es mala porque se pusieron bravos". "Si yo soy victima [sic] me pusiera [sic] bravo, porque si me pegan, me desquito, le doy más duro". Esto evidencia que los estudiantes, en su mayoría, al analizar la situación de conflicto presentan dificultad para identificar las partes constitutivas de este; no extraen la idea central y no comprenden la situación; además, en el proceso reflexivo se les dificulta plantear soluciones asertivas y razonables.

Al comparar los hallazgos de las fases uno y dos se pudo evidenciar cómo, a partir del análisis de dilemas y demás acciones didácticas que desarrollaron los estudiantes en la etapa dos, el porcentaje de estudiantes que se ubicó en nivel bajo de análisis descendió, los estudiantes lograron avanzar y ubicarse 
Tabla 1. Niveles de análisis, síntesis y reflexión

\begin{tabular}{|c|c|c|c|}
\hline Habilidades & Nivel bajo & Nivel medio & Nivel alto \\
\hline Análisis & $\begin{array}{l}\text { En el proceso de análisis emite } \\
\text { razones, pero no las justifica } \\
\text { con argumentos razonables y } \\
\text { no logra identificar las partes } \\
\text { que estructuran un conflicto } \\
\text { interpersonal. }\end{array}$ & $\begin{array}{l}\text { En el proceso de análisis } \\
\text { logra dar algunas razones y } \\
\text { las sustenta con argumentos } \\
\text { razonables a partir del reco- } \\
\text { nocimiento parcial de los } \\
\text { elementos de la situación } \\
\text { de conflicto. }\end{array}$ & $\begin{array}{l}\text { En el proceso de análisis } \\
\text { logra dar argumentos ra- } \\
\text { zonables a partir de la des- } \\
\text { composición y la relación } \\
\text { organizada de los elementos } \\
\text { constitutivos de la situación } \\
\text { de conflicto. }\end{array}$ \\
\hline Síntesis & $\begin{array}{l}\text { En el proceso de síntesis no } \\
\text { acude a las partes elementales } \\
\text { del conflicto y a su interrela- } \\
\text { ción para comprender la situa- } \\
\text { ción. No presenta argumentos } \\
\text { razonables. }\end{array}$ & $\begin{array}{l}\text { En el proceso de síntesis } \\
\text { presenta argumentos razo- } \\
\text { nables acudiendo a algunos } \\
\text { elementos constitutivos de la } \\
\text { situación de conflicto y las } \\
\text { une para su comprensión. }\end{array}$ & $\begin{array}{l}\text { En el proceso de síntesis } \\
\text { emite un razonamiento } \\
\text { acudiendo a las partes ele- } \\
\text { mentales de la situación de } \\
\text { conflicto y las relaciona de } \\
\text { forma organizada para su } \\
\text { comprensión. }\end{array}$ \\
\hline Reflexión & $\begin{array}{l}\text { En el proceso de reflexión el } \\
\text { estudiante expone su punto de } \\
\text { vista y una posible solución, } \\
\text { pero no lo sustenta con argu- } \\
\text { mentos razonables. }\end{array}$ & $\begin{array}{l}\text { Evalúa la situación de con- } \\
\text { flicto y emite una posible } \\
\text { solución. Intenta sustentar } \\
\text { su posición sin mayor jus- } \\
\text { tificación. }\end{array}$ & $\begin{array}{l}\text { A partir de los procesos de } \\
\text { análisis y síntesis evalúa } \\
\text { la situación, plantea una } \\
\text { posible solución asertiva y } \\
\text { autónoma al conflicto y la } \\
\text { sustenta con argumentos } \\
\text { razonables. }\end{array}$ \\
\hline
\end{tabular}

Fuente: elaboración propia.

en un nivel medio al finalizar la etapa dos. En cuanto al proceso de síntesis, el nivel bajo se redujo y los estudiantes lograron avanzar a un nivel medio. En los procesos reflexivos, el nivel bajo se redujo a la mitad y se pudo identificar la evolución a un nivel medio. Como se puede evidenciar, cuando se le pregunta al estudiante E6: "iqué opinas de lo que hizo Diego?" o "itú qué harías en el lugar de Juan?, ¿por qué lo harías?, respondió: "opino que lo que hizo es malo, porque el [sic] no tuvo valor de entregar el bolso, amenazo [sic] a Juan, le puso el bolso a José". "Yo haría: avisarle a la profesora y si me amenaza le digo a la rectora para que hablen con Diego, lo haría para que solucionen el problema y hablen con él y para que mejoren las actitudes de Diego". En esta fase, al realizar el proceso de análisis, síntesis y reflexión, los estudiantes resaltan que se debe abordar un conflicto con la ayuda de un adulto.

En la fase tres el progreso fue más significativo: los estudiantes lograron avanzar a un nivel de análisis alto; en el proceso de síntesis el nivel bajo se redujo, ya que los estudiantes se mantuvieron en 
$\overline{\text { Angélica María Rodríguez Ortiz, Rosa Elmira Coral Cadena, María Guadalupe Andino, Óscar William Portilla }}$

nivel medio y consiguieron pasar a un nivel alto; por último, en los procesos de reflexión el avance fue revelador: la mayoría de los estudiantes logró avanzar a un nivel alto.

La contrastación de estos resultados permite manifestar que los estudiantes fueron fortaleciendo de manera gradual sus procesos de análisis, síntesis y reflexión, y esto se vio reflejado en los procesos argumentativos; así lo muestran algunas de sus respuestas: "yo opino que está mal porque al no decir la verdad culpan a Mauricio de algo que el no hizo"; "la mejor decisión es que los protagonistas deben dialogar porque es la manera más justa de solucionar un conflicto"; "porque es el diálogo la mejor forma para que ellos puedan entender lo que pasó y llegar a un acuerdo y así solucionen los problemas". En sus planteamientos denotaron elementos argumentativos más coherentes y contextualizados que sustentan posibles soluciones razonadas y razonables a las situaciones conflictivas presentadas en los dilemas morales; "[...] las soluciones deben resultar de una discusión, un diálogo, una argumentación, en donde las distintas opiniones tengan la oportunidad de salir a la luz y ser confrontadas entre sî" (Haba, 1978, p. 21).

Estos resultados señalan la relevancia y el impacto de habilidades en la mejora de los argumentos y la pertinencia 44 de emplear dilemas morales; puesto que estos permitieron a los estudiantes alcanzar una mejor comprensión del conflicto y el planteamiento de posi- bles soluciones razonadas y razonables sustentadas desde una perspectiva constructiva; adicional a esto, vincularon procesos argumentativos que fueron perfeccionando e hicieron un mejor uso de formas argumentativas como la razón, la garantía y la conclusión (que son algunos de los modelos propuestos por Toulmin [2007]).

\section{Modelos argumentativos}

Para evaluar los cambios y transformaciones en los estudiantes fue necesario analizar sus formas argumentativas; para ello se utilizaron algunos modelos argumentativos que propone Toulmin (2007), como la razón (implícita en los datos), el uso de garantías y conclusiones. Las conclusiones surgen de los datos y se apoyan en las garantías, y las hacen más convincentes. El uso de las garantías permite evaluar si la aserción se basa en la evidencia, siendo el puente desde el cual ambos dependen. Según Toulmin (2007): “[... L La garantía funciona justamente como puente necesario para realizar la transición de los datos a la conclusión" (p. 188).

Se encontró en la primera fase que los estudiantes usaron razones causales para justificar las formas disruptivas que venían aplicando en el abordaje de un conflicto, como se evidencia en el ejemplo: "El problema es el maltrato hacia los niños más pequeños. Yo opino que está muy mal porque ellos en vez de tratar de solucionar el problema lo empeoraron y pelearon" y las usaron para tratar de justificar que un conflicto es malo 
y que, por tanto, afecta las relaciones mismas. Lo anterior también se puede evidenciar cuando ellos manifiestan: "La forma como abordaron fue mala, porque quedaron bravos entre ellos". Esto lo sustenta Toulmin (2007), cuando dice que "[...] las razones causales expresan el motivo por el cual algo ha ocurrido o habrá de ocurrir, sus efectos" (Toulmin, citado en Rodríguez, 2004, pp. 7 y 8).

Por otra parte, se puso en evidencia que, en su mayoría, los estudiantes no hacen uso de garantía, como se constata en los ejemplos: "La relación entre esos compañeros es muy mala porque no se comparten sus cosas por envidia"; "yo la primera vez le diría que no me moleste y si me sigue molestando yo le pegaría y me pondría muy brava". Por las razones expuestas más arriba, la ausencia de garantías no permite dar una transición coherente a la conclusión. Según Toulmin (2007), si no se percibe la fuerza de los argumentos, entonces la conclusión es poco plausible e incomprensible para cualquier receptor que lo analice. Esto denota que en los argumentos los estudiantes presentan una concepción negativa del conflicto. Para Toulmin (2007), la garantía permite evaluar si la aserción se basa en la evidencia: "[...] la garantía funciona justamente como puente necesario para realizar la transición de los datos a la conclusión" (p. 188).

Respecto al uso de la conclusión, la mitad de los estudiantes develaron argumentos incoherentes, ya que usaron premisas contradictorias. Presentaron incongruencia entre la garantía y la conclusión, incompatibilidad entre los datos y la conclusión o una transición incoherente desde los datos hasta la conclusión. Al posicionarse los estudiantes en el papel de protagonistas, se puede notar que sus planteamientos no se sustentan en argumentos razonados y razonables. Así lo demuestran cuando manifiestan que: "Los personajes se sentían con rabia. Opino que estuvo mal, porque no dialogaron"; "actuaría mal porque me sacan la rabia". Esto se relaciona con lo que plantea Toulmin (2007): "Una conclusión desnuda, desprovista de datos que la apoyen, no es un argumento" (pp. 143 y 144). Lo anterior ratifica que los estudiantes no sustentan sus planteamientos con argumentos; no cuentan con habilidades que les permitan proponer posibles soluciones a los conflictos interpersonales.

Los estudiantes que plantearon como posible solución la mediación de un adulto también mostraron conclusiones incongruentes con los datos y las garantías que usaron; fueron pocos los que se aproximaron a un abordaje un poco más asertivo, pero no mostraron el uso de formas argumentativas para sustentar sus planteamientos. Se evidenció la concepción negativa que tienen del conflicto; no lograron hacer uso de formas argumentativas para proponer una posible solución autónoma y justificada con premisas razonables.

Por otra parte, en la etapa dos el uso de los modelos argumentativos se transforma; 
$\overline{\text { Angélica María Rodríguez Ortiz, Rosa Elmira Coral Cadena, María Guadalupe Andino, Óscar William Portilla }}$

los estudiantes dan un paso significativo porque usaron razones causales para evaluar las consecuencias y juzgar el mal manejo que los involucrados dan a los conflictos interpersonales, como lo señala un estudiante: "Opino que lo que hizo es malo, porque él no tuvo el valor de entregar el bolso, amenazó a Juan, le puso el bolso a José". Teniendo en cuenta los aportes de Toulmin (2007), las razones son "[...] los elementos justificatorios que alegamos como base de la afirmación realizada" (p. 133). Como se puede ver en una de las respuestas, los estudiantes proponen motivos para defender sus argumentos; los restantes no lograron hacer buen uso de este elemento, pues con sus respuestas no justifican por qué un conflicto es perjudicial y daña las relaciones.

Por otro lado, se pudo observar que los estudiantes usaron garantías para legitimar el paso de los datos a la conclusión. Se hace uso de garantías implícitas (decir la verdad), como lo corrobora uno de los estudiantes: "Yo haría avisarle lo que pasó a la profesora". Estos argumentos dan cuenta de que los estudiantes fundamentan sus propuestas desde un abordaje constructivo del conflicto interpersonal. Según Toulmin (2007), una garantía "[...] indica la fuerza que posee el apoyo que motiva la aserción" (p. 125). No obstante, una minoría no hizo uso de garantías, porque sus ar46 gumentos no están lo suficientemente relacionados con sus aseveraciones; no permiten una transición coherente a sus conclusiones, pues en estas se evidenció incongruencia entre los datos y la conclusión por el uso de premisas contradictorias. "La garantía es una consecuencia general de carácter práctico sobre la manera en que se puede argumentar con seguridad a la vista de esos hechos" (Toulmin, 2007, p. 143).

En la etapa 2 se identificaron las características de sus argumentos. Se encontró que hay mayor coherencia entre los datos y la conclusión, pues las premisas tienen mayor coordinación. Las conclusiones se apoyaron en las garantías cuando intentaron justificarlas; utilizaron razones aproximándose a argumentos razonados y razonables a una solución asertiva al conflicto. A partir de los resultados, se puede evidenciar que algunos estudiantes trataron de inclinar sus planteamientos hacia lo que se propone en sus conclusiones. Se vio un abordaje autónomo, aunque faltó aún más fuerza argumentativa, como se demuestra cuando responden: "[...] si me amenaza le digo a la rectora para que hablen con Diego, lo haría para que solucionen el problema con él y para que mejoren las actitudes de Diego". Aquí la conclusión se reafirma con la garantía, y así se solidifica al argumento desde sus premisas; así, su objetivo es "[...] mostrar cómo a partir de esos datos hemos pasado a la afirmación original o conclusión y que el paso de los primeros a la segunda es apropiado y legítimo" (Toulmin, 2007, p. 134). Sin embargo, solo un grupo reducido planteó posibles soluciones asertivas (aunque mostraron inconsistencia en sus 
conclusiones y deficiente argumentación en sus planteamientos).

En la fase tres se hizo evidente un mejor uso de estos tipos de argumentos; un gran número de estudiantes usaron las razones causales para sustentar formas asertivas y razonables de abordar un conflicto, como se puede evidenciar en los siguientes ejemplos: "David debe tener el valor de decirle la verdad al rector, y también a Camilo, porque es que él no dialogó sobre el tema" y "el comportamiento de David fue malo, se asustó y se fue para los baños a esconderse y no dijo la verdad, por eso culpan a Mauricio. La actitud fue mala, porque él no quería asumir las consecuencias". Según Toulmin, "[...] las razones (grounds) son aquellas proposiciones que se aportan a la hora de defender la tesis" (citado en Cardona, 2008, p. 31). Al final del proceso los estudiantes hacen uso de razones causales para proponer y defender posibles soluciones desde una visión constructiva del conflicto; con estas soluciones sustentan un abordaje mediado por el diálogo, la construcción de acuerdos y la toma de decisiones justas para las partes en conflicto.

Al hacer seguimiento al uso de garantías también se corroboró un gran avance. En esta fase se identificó que los estudiantes hacen mayor uso de garantías; fue evidente el uso de este modelo en sus argumentos para reafirmar y dar más fuerza argumentativa a sus conclusiones. "Las garantías corresponden a los estándares prácticos o cánones de argumentos" (Toulmin, 2007, p. 134). Según esto, la garantía permite que haya legitimidad y coherencia entre los datos y la conclusión. Para Toulmin (2007), una garantía "[...] confiere diversos grados de fuerza a las conclusiones que justifican" (p. 137).

En la fase 3 el análisis puso de manifiesto que solo una minoría de estudiantes reveló conclusiones sin mayor justificación. En la mayoría de los casos prevalece la mediación como forma de solucionar el conflicto interpersonal y gran parte de los estudiantes logró emitir conclusiones en las que reflejan una mejor estructuración de los argumentos, una relación coherente de las razones y garantías, y un mejor uso del lenguaje, con el propósito de sustentar posibles soluciones enfocadas hacia una perspectiva constructiva del conflicto. En la figura 2 se evidencia un argumento basándose en la estructura argumentativa de Toulmin (2007).

\section{Categoría conflicto interpersonal}

Al presentar los hallazgos encontrados en esta categoría - y para mayor comprensión- se dividió esta en tres subcategorías: conceptualización del conflicto interpersonal, elementos del conflicto interpersonal y abordaje del conflicto interpersonal.

En la fase uno y dos se evidenció que los estudiantes presentaron obstáculos epistémicos, lo que dificulta la apropiación del conocimiento. En la subcategoría conceptualización del conflicto 


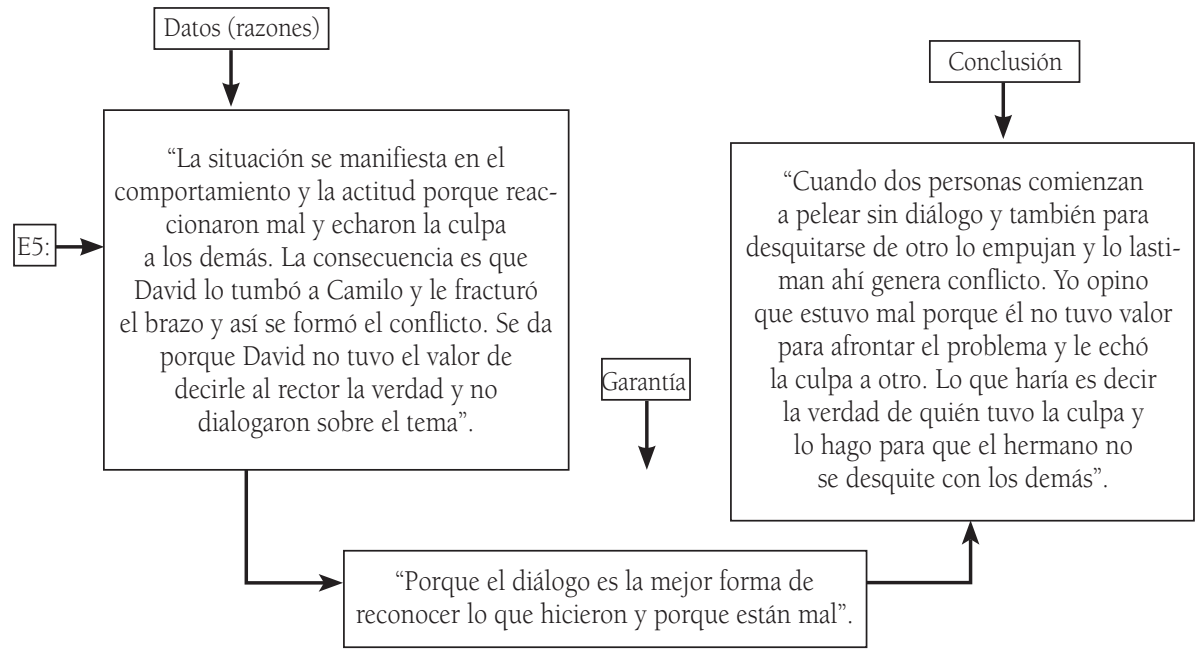

Figura 2. Modelos argumentativos utilizados por los estudiantes

Fuente: elaboración propia.

interpersonal, se encontró que la mayoría de estudiantes conceptualizan el conflicto como negativo, es decir, como un problema que no tiene solución. Esto se evidencia en una de las respuestas más recurrentes de los estudiantes: "Para mí un conflicto interpersonal son las peleas que hay entre compañeros. El conflicto es malo, porque no nos deja vivir en paz y armonía". Acá se pone de manifiesto que los niños poseen obstáculos al comprender el concepto de conflicto interpersonal. Ante esto, la Fundación UNIR Bolivia (s. f.) hace referencia a que "[... el el conflicto en una visión destructiva significa amenaza o enfrentamiento" (p. 12).

En la fase tres los estudiantes demuestran gran cambio en sus connotaciones hacia el conflicto interpersonal, y lo enfocan en una perspectiva constructiva y hacia el concepto de conflicto como oportunidad de aprendizaje que fortalece las relaciones, como lo señala un estudiante al responder: "[...] Con el conflicto aprendemos a ponernos en el lugar del otro y así entenderlos mejor y poder tener una relación amistosa y mantenernos en armonía”.

En la subcategoría elementos del conflicto interpersonal, gran parte de los estudiantes no identificaron dichos elementos claramente. Así, como se evidencia en E1: "Por lo que forma el conflicto, la persona que forma el problema”. Ante esto, Lederach (1993) dice: "Es necesario, en primer lugar, analizar y comprender la naturaleza, estructura y elementos que componen todo conflicto" (p. 137). 
Por otra parte, en la fase dos los estudiantes lograron identificar la contradicción como causales del conflicto y en la fase tres, los elementos estructurales de este. El análisis mostró que coincidieron con el E5, cuando este manifiesta que:

Los elementos que contiene un conflicto interpersonal son causas, personajes y consecuencias esto debe conocerse, para así no culpar a un inocente. Las causas nos sirven para solucionar adecuadamente los conflictos. Las consecuencias nos sirven para pensar antes de actuar. Los conflictos interpersonales se dan por las diferentes creencias, las costumbres, la falta de respeto y por las formas de pensar de los demás compañeros.

En la subcategoría abordaje del conflicto interpersonal, la mayor parte de los estudiantes coinciden en que se actúa agresivamente, como lo demuestra el E3: "Actuamos con insultos, con peleas verbales o físicas, actuamos de esa manera porque nos da rabia y nos ponemos bravos, porque no dejan vivir en paz y armonía"; esto muestra que abordan el conflicto mediante formas disruptivas. Otros estudiantes asumen un rol complaciente o un rol evasivo, porque, según Yánez y Galaz (2011), "[...] los conflictos inadecuadamente resueltos o la agresividad natural no controlada pueden derivar en situaciones de violencia" (p. 36). En la tercera fase los estudiantes proponen la mediación de un tercero que les ayude a resolver una la situación conflictiva.
En la fase tres se evidenció que los estudiantes plantean argumentos más coherentes y razonables, como lo corrobora el E13: "Forma de abordarlo: es ponernos de acuerdo con las ideas que pensamos diferentes. La manera más adecuada es analizando, dialogando con mi compañero para que no siga el conflicto y tener en cuenta que en el conflicto hay cosas positivas para que haya paz".

Los resultados mostraron un cambio significativo en las formas como argumentan los estudiantes en el momento de plantear soluciones razonadas y razonables a los conflictos interpersonales. Esto hace aún más consistente la problemática de estudio que permitió comprender el conflicto desde sus elementos constitutivos y desarrollar habilidades a través de la argumentación.

\section{Categoría niveles de desarrollo moral}

Para poder comprender más a fondo los planteamientos de los estudiantes fue necesario el análisis de sus niveles de desarrollo moral, porque definen la capacidad para razonar moralmente y de actuar con autonomía responsable. Según lo enuncian Rodríguez y Hernández (2016), apoyados en la teoría de Kohlberg: "[...] De acuerdo con las etapas de desarrollo, surgen diferencias en las formas de pensar de los niños de edades diferentes. Así mismo, también se manifiestan diferencias en las formas de actuar" (p. 35). 
Angélica María Rodríguez Ortiz, Rosa Elmira Coral Cadena, María Guadalupe Andino, Óscar William Portilla

En la fase uno, de acuerdo con las características de los argumentos de los estudiantes, se encontró que la mayoría se encuentra en un nivel preconvencional (etapa de orientación heterónoma) que, según Kohlberg (1992), es la etapa donde el sujeto toma decisiones y moldea su comportamiento, tomando como base las normas que le impone una autoridad, y asume una actitud de obediencia por temor al castigo. De estos, algunos se ubicaron en la etapa autoritaria porque, según Kohlberg (1992), la persona que se encuentra en esta etapa tiende a la obediencia por miedo al castigo y reconoce la autoridad del adulto: "Evitar el castigo y el poder superior de las autoridades" (p. 188). Por otro lado, algunos estudiantes se ubican en la etapa hedonista porque, según Kohlberg, las personas hedonistas se rigen a "[...] seguir las normas solo cuando es en inmediato interés de alguien" (p.188). En esta etapa se comprende que se actúa por beneficio propio $y$ que pueden entrar en un conflicto con los demás. Por último, una minoría se ubica en el nivel convencional, en la etapa convencionalista. Según Kohlberg (1992), las razones para actuar bien se basan en "[...] la necesidad de ser una buena persona ante uno mismo y ante los demás (p. 188). En esta etapa los niños empiezan a reconocer que sus acciones pueden afectar a los demás y aceptan las normas impuestas por la autoridad. Todo esto se puede evidenciar en las respuestas que dan los niños.
En la fase dos los estudiantes, en su mayoría, se ubicaron en la etapa autoritarista y en la etapa hedonista, y un grupo menor de estudiantes logró avanzar al nivel convencional, a la etapa convencionalista. Sin embargo, ya en la fase tres se pudo observar una reducción más notable del porcentaje de estudiantes ubicados en nivel preconvencional; se visualizó un mínimo de estudiantes que se mantienen todavía en la etapa hedonista y un gran número que logró avanzar al nivel convencional; de esta manera, se ubicaron en las etapas convencionalista y consecuencialista. ${ }^{2}$

Al poner en marcha procesos argumentativos y de razonamiento, y al comparar el desempeño en los niveles de desarrollo moral, es posible afirmar que los estudiantes avanzan gradualmente

2 Es preciso aclarar que, aunque la teoría de Kohlberg contempla seis estadios y en este caso los niños solo alcanzan los dos primeros, como lo plantean Rodríguez y Hernández (2016), solo muy pocos adultos llegan a alcanzar el estadio deontológico y, con más razones, es muy poco probable que un niño alcance tal nivel. Sin embargo, sí es necesario hacer una estimulación temprana en el desarrollo de estos estadios a una corta edad para que, de esta manera, existan más posibilidades de que a futuro se forme un hombre deontológico. Que algunos niños en tan solo tres fases hayan conseguido avanzar un estadio, que otros pocos hayan avanzado dos y que incluso otros hayan avanzado tres es un resultado relevante si se tiene en cuenta la exigencia de cada paso y que, además, como lo indican Rodríguez y Hernández (2016), la mayoría de los adultos nunca logran superar el primer estadio (p. 41). 
a un nivel superior y, a su vez, que en la fase tres aumentan significativamente al nivel convencional. Así lo constata el E4 al manifestar que cuando se aborda una situación conflictiva se debe "Hablar con él y preguntarle por qué me rempujo y me hizo lisiar el brazo". "Debemos hablar y dialogar para solucionar los problemas para seguir siendo amigos" (etapa convencionalista). Con este planteamiento se puede constatar que "[...] el nivel convencional difiere del preconvencional porque utiliza las siguientes razones: 1- Interés por la aprobación social. 2- Interés por la lealtad a las personas, grupo y autoridad. 3- Interés por el bienestar de otros y la sociedad" (Kohlberg, 1992, p. 190).

Este avance es muy significativo, si se tienen en cuenta las características que presentaba el grupo al inicio del proceso: el comportamiento, sus actitudes y sus razonamientos frente a una situación de conflicto no eran los más adecuados.

\section{Conclusiones}

La investigación permitió a los estudiantes reconocer las dificultades y obstáculos en la formulación de argumentos y solución de conflictos interpersonales presentados en el aula, ya que no planteaban soluciones razonadas, sino que se presentaban escenas de intolerancia, agresiones físicas y verbales, como una salida inicial. Colocando a prueba los aprendizajes adquiridos en las fases de la unidad didáctica, se pudo constatar que desde el desarrollo de las habilidades para la argumentación —específicamente el análisis, la síntesis y la reflexión-, a través del análisis de dilemas morales adaptados a la estructura de Kohlberg (1992), los estudiantes lograron acceder a un conocimiento estructural del conflicto, comprender su naturaleza y argumentar un abordaje constructivo proponiendo alternativas razonadas y razonables para la solución de los conflictos interpersonales.

Se puede considerar que para plantear posibles alternativas de solución a los conflictos interpersonales que se presentan en el entorno escolar es necesario realizar un análisis estructural del conflicto, reconocer los protagonistas para disentir sobre los puntos controversiales y llegar a la construcción de acuerdos y a la toma de decisiones justas a través de la argumentación. Estimar las consecuencias fue esencial para que los estudiantes reflexionaran críticamente sobre los efectos que pueden tener sus actos para los antagonistas un conflicto resuelto de forma destructiva y uno de manera constructiva.

El proceso de síntesis y representación del conflicto les permitió identificar la idea central, y acudir así a sus elementos constitutivos y a la cohesión que se da entre estos para evaluar su dimensión y anticipar los resultados. En función de los datos extraídos, los estudiantes accedieron a su comprensión y lograron emitir argumentos coherentes y razonables que sustentaron una solución constructiva del conflicto. 
$\overline{\text { Angélica María Rodríguez Ortiz, Rosa Elmira Coral Cadena, María Guadalupe Andino, Óscar William Portilla }}$

El proceso de reflexión permitió evaluar el abordaje del conflicto, examinar sus consecuencias, canalizar la generación de planteamientos coherentes y asertivos para proponer un abordaje cooperativo y negociador, y el planteamiento de posibles soluciones al conflicto interpersonal. Esto suscitó un proceso de reflexión que los llevó a tener una perspectiva frente al rol de los protagonistas para decidir cómo y por qué deben actuar de esa manera. Así, se vieron en la necesidad de sustentar sus propuestas con razones y de incluir garantías para apoyar lo que a su juicio moral les parece más correcto. Se resalta, entonces, que desde estos procesos se impulsa el uso de algunos modelos argumentativos (razón, garantía y conclusión) propuestos por Toulmin (2007), los cuales fortalecieron los planteamientos de los estudiantes, y les dieron más fuerza y coherencia argumentativa.

Este proceso enfrentó a los estudiantes hacia el uso de formas argumentativas y propició el razonamiento moral, aspecto que favoreció el planteamiento de posibles soluciones razonadas y razonables. Con lo anterior se logró una conceptualización del conflicto interpersonal desde una perspectiva constructiva.

Un abordaje asertivo de las situaciones conflictivas propicia el desarrollo de las habilidades para la argumentación que, a su vez, posibilita a los estudian52 tes la evaluación crítica de sus propios planteamientos. Los estudiantes son capaces de exponer sus puntos de vista, justificarlos y defender sus posiciones ante los conflictos, de tal manera que no sea solo para persuadir, sino también para llegar a consensos.

El análisis de dilemas morales utilizados como estrategia en la aplicación de la unidad didáctica fue eficaz para involucrar a los estudiantes en el ejercicio de usar formas argumentativas - como las razones, las garantías y las conclusiones-, lo que favoreció la emisión de razonamientos coherentes y justificados desde un razonamiento moral. Así, fue posible desarraigar la concepción negativa del conflicto y las medidas competitivas y autoritaristas que plantearon los estudiantes al inicio del proceso.

Aproximarse al razonamiento moral de los estudiantes permite comprender su comportamiento y sus planteamientos ante las posibles soluciones a los conflictos interpersonales. En ese sentido, se resalta la importancia de anclar el desarrollo moral en los procesos relacionados con el manejo de conflictos interpersonales, porque estos condicionan la forma de pensar y de actuar. Un nivel de desarrollo moral avanzado permite al estudiante asumir un criterio moral que le conduce a plantear posibles soluciones más asertivas y justas para, al enfrentar un conflicto, actuar con autodeterminación y con racionalidad.

Por todo lo anterior, es imprescindible ofrecer estrategias como el desarrollo de la argumentación, para que los estudiantes justifiquen sus planteamientos 
y puedan fortalecer su desarrollo moral al plantear posibles soluciones, y de esta manera transformar constructivamente el conflicto en igualdad de oportunidades.

\section{Referencias}

Alzate, L. y Henao, F. (2012). Resolución de conflictos en un colegio público Bogotano a través del desarrollo del juicio moral (Tesis de maestría). Universidad de la Sabana, Chía, Colombia.

Bascón, M., De la Mata, M. y Cala, M. (2006). Género, argumentación y resolución de conflictos en adolescentes. Análisis a través del discurso (Tesis doctoral). Universidad de Sevilla, España.

Bermúdez, A. y Jaramillo, R. (2000). El análisis de dilemas morales: una estrategia pedagógica para el desarrollo de la autonomía moral. Bogotá: Secretaria de Educación. Recuperado de http:// files.asambleadeaula.webnode. es/200000629-3c0713dfb0/el_an \%C3\%Allisis_de_dilemas_morales_ una_estrategiia_pedag\%C3\%B3gica_ para_el_desarrollo_de_la_autonom \%C3\%ADa_moral.pdf

Camps, A. y Dolz, J. (1995). Enseñar a argumentar: un desafío para la escuela actual. Comunicación, Lenguaje y Educación, 26, 5-8. Recuperado de https://dialnet.unirioja.es/descarga/ articulo/2941554.pdf

Cardona Rivas, D. (2008). Modelos de argumentación en ciencias: una aplicación a genética (Tesis de doctorado). Centro de Estudios Avanzados en Niñez y Juventud alianza de la Universidad de Manizales y Fundación Centro Internacional de Educación y Desarrollo Humano [CIND], Manizales, Colombia. Recuperado de http://biblioteca.clacso. edu.ar/Colombia/alianza-cindeumz/20130225064303/tcardona.pdf

Cortina, A. (1997). Resolver conflictos, hacer justicia. Cuadernos de Pedagogía, 257, 5456. Recuperado de http://d-f.scribdassets. com/docs/84mljruibk3w5rss.pdf.

Fundación UNIR Bolivia. (s. f.). Guía en capacitación en transformación del conflicto. Recuperado de: http://d-f.scribdassets. com/docs/trhcvrocglogfqa.pdf

Galtung, J. (1981). Hacia una definición de la investigación sobre la paz. En Unesco (Ed.), Investigación sobre la paz. Tendencias recientes y repertorio mundial. París: Unesco.

Haba, E. (1978). Lo racional y lo razonable. Revista de Filosofía de la Universidad de Costa Rica, 16(43), 1-32. Recuperado de http://www.inif.ucr.ac.cr/ recursos/docs/Revista\%20de $\% 20$ Filosof\%C3\%ADa\%20UCR/Vol.\%20 XVI/No.\%2043/lo\%20racional\%20 y\%20lo\%20razonable.pdf

Jares, R. J. (1997). El lugar del conflicto en la organización escolar. Revista Iberoamericana de Educación, 15. Recuperado de http://www.rieoei.org/oeivirt/rie15a 02.htm

Kohlberg, L. (1992). Psicología del desarrollo moral (Trad. A.Zubiaur Zárate). Bilbao: Descleé de Brouwer, S. A.

Lederach, J. P. (1993). Elementos para la resolución de conflictos. Recuperado de http://es.slideshare.net/teoriadelaconciliacion/lederach 
$\overline{\text { Angélica María Rodríguez Ortiz, Rosa Elmira Coral Cadena, María Guadalupe Andino, Óscar William Portilla }}$

Migdalek, M., Santibáñez, C. y Rosemberg, C. (2014). Estrategias argumentativas en niños pequeños: un estudio a partir de las disputas durante el juego en contextos escolares. Signos, 47(86), 435462. Recuperado de http://www.scielo. cl/scielo.php?script=sci_arttext\&pid =S0718-09342014000300005

Ministerio de Educación Nacional (MEN) (2014). Guía pedagógica. Manual de convivencia. Colombia Aprende. Recuperado de http:// www.colombiaaprende.edu.co/html/ micrositios/1752/articles-340940_ archivo_pdf.pdf

Monsalve Upegui, M. E. (2015). Estado del arte de la investigación sobre argumentación y escritura multimodal desde una perspectiva didáctica. Revista Lasallista de Investigación, 12(2), 215 224. Recuperado de http://www.scielo. org.co/pdf/rlsi/v12n2/v12n2a22.pdf

Perales, F. y Cañal de León, P. (2015) Didáctica de las ciencias experimentales. Barcelona: Marfil.

Perelman, C. y Olbrechts-Tyteca, L. (1989). Tratado de la argumentación, la nueva retórica. (Trad. J. Sevilla Muñoz). Madrid: Gredos.
Rodríguez, A. y Hernández, J. (2016). La filosofía y la formación moral en la actualidad. Saarbrücken: Editorial Académica Española.

Rodríguez, L. (2004). El modelo argumentativo de Toulmin en la escritura de artículos de investigación educativa. Caracas: Universidad Pedagógica Experimental Libertador.

Ruiz, A. y Chaux E. (2005). La formación de competencias ciudadanas. Bogotá: Asociación Colombiana de Facultades de Educación [Ascofade].

Sánchez, L., González J. y García A. (2013). La argumentación en la enseñanza de las ciencias. Revista Latinoamericana de Estudios Educativos, 1(9), 11-28.

Toulmin, S. (2007). Los usos de la argumentación (Trad. M. Morras y V. Pineda). Barcelona: Península.

Ugalde, I. (2009). Resolución de conflictos en la educación infantil (Tesis de maestría). Universidad de Barcelona, España.

Yánez, P. y Galaz, J. (2011). Conviviendo mejor en la escuela y en el liceo. Orientaciones para abordar la convivencia escolar en las instituciones educativas. Chile: Ministerio de Educación. 